\title{
The Next Level Implant Biomaterial: Silicon Nitride
}

\author{
Arpit Sikri ${ }^{1 *}$, Jyotsana Sikri \\ ${ }^{1}$ Senior Lecturer, Department of Prosthodontics, Santosh Dental College, Santosh Deemed to be University, \\ Ghaziabad, Delhi NCR, India \\ ${ }^{2}$ Senior Lecturer, Department of Conservative Dentistry and Endodontics, Santosh Dental College, Santosh \\ Deemed to be University, Ghaziabad, Delhi NCR, India
}

*Corresponding Author: Arpit Sikri, Senior Lecturer, Department of Prosthodontics, Santosh Dental College, Santosh Deemed to be University, Ghaziabad, Delhi NCR, India; Email: arpitsikri@gmail.com

Received Date: 28-10-2020; Accepted Date: 19-11-2020; Published Date: 27-11-2020

Copyright $^{\complement} 2020$ by Sikri A, et al. All rights reserved. This is an open access article distributed under the terms of the Creative Commons Attribution License, which permits unrestricted use, distribution, and reproduction in any medium, provided the original author and source are credited.

\section{Editorial}

Dental caries and periodontal disease form the two most important factors for tooth loss. Rehabilitation of the lost dentition involves either fixed or removable prosthesis. Above all, dental implants have also proven to be the 1st choice of treatment in terms of prosthetic rehabilitation. According to glossary of Prosthodontic Terms ( $9^{\text {th }}$ Edition), dental implant is defined as a prosthetic device made of alloplastic material(s) implanted into the oral tissues beneath the mucosal and/or periosteal layer and on or within the bone to provide retention and support for a fixed or removable dental prosthesis; a substance that is placed into and/or on the jaw bone to support a fixed or removable dental prosthesis. In addition to this, dental implant is that portion of an implant which provides support for the dental implant abutment(s) through adaptation on (eposteal), within (endosteal), or through (transosteal) the bone.

Any substance other than a drug that can be used for any period of time as part of a system that treats, augments, or replaces any tissue, organ, or function of the body is known as a biomaterial. A plethora of materials have been used as a dental implant material namely titanium alloys, zirconia, and polymers. Recently, a variety of biomaterials including metals, ceramics, and polymers have been explored for the fabrication of core dental implant and surface coatings. Titanium has always been used as a gold standard in dental implant biomaterials. However, titanium has proven to be a "Double Edge Sword". This can be attributed to the fact since titanium and its alloys can corrode and wear leading on to deposition 
of the corroded metal particles in the surrounding tissues, thereby leading onto the subsequent inflammation in the nearby area. In due course of time and with the aesthetic demand in patients, this gave birth to the metal free or ceramic dental implants mainly zirconia, which proved to be more biologically inert and plaque resistant comparatively. Furthermore, materials like Polyetheretherk-etone (PEEK) and Silicon Nitride ( $\mathrm{SiN}$ ) have been introduced and tested for implant applications.

The Silicon Nitride $\left(\mathrm{Si}_{3} \mathrm{~N}_{4}\right)$ is an established bioceramic material with exceptional mechanical properties, thermal shock resistance, and low wear resistance making it more suitable for spinal and orthopaedic implants, knee joint replacement, and total hip replacement. In addition, it is also considered for potential dental applications such as dental implants, crowns, and endodontic post core etc. Apart from this properties like passivation, superior antibacterial activity, osseointegration and biocompatibility also form a major part of silicon nitride.

Recently, Silicon nitride is the material of choice for skeletal prosthesis; however, it has been proven an excellent biocompatibility and ultimately proving to be a boon as implant biomaterial in orthopedic implantology. There have a lot of unanswered questions pertaining to implant surface modifications in relation to silicon nitride. The metal-free nature of this material makes it a more promising candidate for dental implantology as the metallic by-products can leach out of the material and invades any part of the body. Higher esthetics, better osseointegration, biocompatible nature, antibacterial property along with high wear resistance and metal free corrosive products make $\mathrm{SiN}$ a good potential candidate for dental implants.

Keeping in view the superior properties and plethora of advantages in relation to silicon nitride, it can be rightly honoured with the statement i.e. "The Next Level Implant Biomaterial". However, further research and clinical trials may further validate in vivo performance of SiN antibacterial surface coatings such as enhancing bone regeneration around the implant surface or reducing the peri-implantitis. 